\title{
Recognition of Multiple Concatenated Arm Gestures using Six-axis Inertial Sensor Signals
}

\author{
Byeong Jeong Kim ${ }^{1}$, Jae-il Jung ${ }^{2}$ and Seop Hyeong Park ${ }^{3 *}$ \\ ${ }^{1}$ Dept. of Electronic Engineering, Hallym University, Chunchon, Korea \\ ${ }^{2}$ Dept. of Electronic Engineering, Hanyang University, Seoul, Korea \\ ${ }^{3}$ Dept. of Electronic Engineering, Hallym University, Chunchon, Korea \\ ${ }^{1}$ kevin@hallym.ac.kr, ${ }^{2}$ jijung@hanyang.ac.kr, ${ }^{3}$ spark@hallym.ac.kr
}

\begin{abstract}
In this paper, we propose a dictionary-based template matching algorithm that can recognize multiple concatenated $3 D$ gestures without detecting the activity period in advance. The proposed algorithm is applied to three-axis gyroscope sensor signal combined with three-axis acceleration sensor signal. We define 14 primitive gestures with different lengths. Every sample of the collected six-dimensional sensor signals is vectorquantized by the k-means clustering algorithm in the six-dimensional vector space. The proposed gesture recognition algorithm uses the Euclidean distance between the templates in the gesture dictionary and the input data. To evaluate the recognition accuracy of the proposed algorithm, we experimented with changing the length of the rest area between gestures. As a result of 14 primitive gesture recognition tests with the proposed algorithm, the overall recognition accuracy of $96 \%$ or more was obtained regardless of the length of the pause period between two adjacent gestures.
\end{abstract}

Keywords: gesture recognition, IMU sensor, human-machine interaction, clustering

\section{Introduction}

With the development of computers and smart devices, interest in designing interfaces between people and computers has increased. There has been a strong tendency for Human Computer Interaction (HCI) to pursue a more intuitive and easy-to-use interface. Because gestures can be highly effective non-verbal means to convey meaning, gesture recognition technology has been extensively studied as a tool of $\mathrm{HCI}$ in many fields such as device manipulation, game, etc. [1-5].

Gesture recognition techniques are divided into three categories according to sensor types: data glove-based gesture recognition, computer vision-based gesture recognition, and sensor-based gesture recognition. In recent years, various methods of recognizing gestures based on bio-signals by attaching numerous sensors to a part of the body have been studied [6-10], whereas a variety of devices have been commercialized and proven to be practical.

In order to increase the recognition rate of various gestures, different sensors such as camera, Electromyography (EMG), and Inner Measure Unit (IMU) sensors are combined to perform gesture recognition like with Kinect [11] and Myo Armband [12].

The data glove-based gesture recognition technology, as the name implies, uses a glove with stretchable bending sensors attached in it. When the user moves the fingers with the glove, the sensors detect the degree of flexion of each finger and determine the data as the user's motion can be estimated from the data. The data glove-based gesture recognition technology senses the degree of finger flexion through the bending sensor attached to the

Received (January 3, 2018), Review Result (March 5, 2018), Accepted (March 12, 2018)

${ }^{*}$ Corresponding Author 
globe and recognizes the movement of the hand through it. This approach is disadvantageous in that it cannot be used for a long time due to the inconvenience of wearing it and the limited movement of the fingers [13].

The computer vision-based method has a disadvantage in that it is difficult to obtain motion information on a three-dimensional space because the image captured by the camera loses depth information in the process of projecting the three-dimensional information onto the two-dimensional plane. In order to obtain depth information, a method of using an RGBD sensor [14] or using an infrared sensor with a camera or using several cameras is also used. However, the disadvantage of limited range of motion and sensitivity to the environment remains a problem.

The sensor-based gesture recognition method can compensate the drawbacks of the two previous methods in that motion information on the three-dimensional space can be obtained and the recognition range is not limited to a large extent. In [15], by combining a three-axis acceleration sensor and an EMG sensor, Xu Zhang et al. have built a system that enables sign language recognition and device control. [16] describes the method of realtime recognition of American sign language (ASL) by combining the signal output from the EMG sensor and the IMU sensor of the wearable armband device. [17] describes a method of recognizing a gesture by encoding the signal output from an acceleration sensor and the gyroscope sensor of a smartphone. The output signal of the IMU sensor is a multidimensional time series signal. The problem of recognizing a gesture from a signal of a specific section of the signal can be considered as a pattern recognition problem. A lot of excellent well-known methods to solve this problem have been exploited, among which are the Hidden Markov models (HMMs)[18], the artificial neural networks[25], and the dynamic time warping (DTW) [17].

Most of the 3D gesture recognition algorithms introduced so far assume that the start and end points of a specific gesture period can be completely detected in advance. Therefore, the overall gesture recognition accuracy depends entirely on the accuracy of the gesture interval detection technique. For this reason, these algorithms only recognize isolated gestures, and it is not easy to recognize consecutive gestures.

In this paper, a 3D gesture recognition method that can recognize multiple concatenated gestures using three-axis acceleration sensor and gyroscope sensor with no need of gesture interval detection in advance is proposed. The proposed method detects the gesture per sample in contrast to the conventional method of detecting the activity periods and recognizing the gesture of the detected interval.

The rest of the paper is organized as follows. In section 2, related work on 3D gesture recognition methods is examined. Section 3 explains the data collection methods and the gesture recognition algorithm proposed in this paper. Section 4 explains the wristwatchtype device and software for training data collection, and compares the performance of the two algorithms for the continuous gesture recognition method proposed in the paper. Finally, conclusions are drawn in Section 5.

\section{Related Work}

In recent years, 3D gesture recognition techniques using IMU have been studied. 3D Gesture classification techniques are classified into three major categories: statistical based approaches, machine learning based classification approaches, and template matching approaches.

Hidden Markov models (HMMs) have been mainly used in statistics-based approaches. HMM is a statistics-based modeling technique for analyzing time-series data and is widely applied to various fields including speech recognition, hand-written character recognition, and gesture recognition. Acceleration sensor-based gesture recognition using the HMM has been studied in [19-23]. Kela et al., used the k-means clustering algorithm to vectorquantize $3 \mathrm{D}$-vector data produced by an accelerometer into 1D-symbols, applying the 
HMMs to recognize the user's hand gesture. If the length of the input data sequence is too long or too short, linear min-max scaling technique was applied to adjust the length of the input sequence before the quantization.

The second approach to 3D gesture recognition is to use machine learning-based classifiers. Hoffman et al. examined two different machine learning algorithms: a simple linear classifier and a classifier based on the AdaBoost framework [24-25].

The last category is based on template matching. In this approach, some representative gesture references for each gesture class are collected to form a collection of templates, and a decision rule for a test gesture is designed. Dynamic time warping (DTW) has shown good performance on the simple time-series pattern recognition problems. The main drawback of this approach is the necessity to find the most appropriate samples to be included in a gesture class while keeping the number of these samples as low as possible to minimize the processing time. Wilson et al. compared the performance of Linear Time Warping and Dynamic Time Warping (DTW) to the HMM-based strategy in [26]. Liu et al. modified the DTW strategy to get more successful results [17].

In recent years, 3D gesture recognition techniques using IMU have been studied. 3D Gesture classification techniques are classified into three major categories: statistical based approaches, machine learning based classification approaches, and template matching approaches.

Hidden Markov models (HMMs) have been mainly used in statistics-based approaches. HMM is a statistics-based modeling technique for analyzing time-series data and is widely applied to various fields including speech recognition, hand-written character recognition, and gesture recognition. Acceleration sensor-based gesture recognition using the HMM has been studied in [19-23]. Kela et al., used the k-means clustering algorithm to vectorquantize 3D-vector data produced by an accelerometer into 1D-symbols, applying the HMMs to recognize the user's hand gesture. If the length of the input data sequence is too long or too short, linear min-max scaling technique was applied to adjust the length of the input sequence before the quantization.

The second approach to 3D gesture recognition is to use machine learning-based classifiers. Hoffman et al., examined two different machine learning algorithms: a simple linear classifier and a classifier based on the AdaBoost framework [24-25].

The last category is based on template matching. In this approach, some representative gesture references for each gesture class are collected to form a collection of templates, and a decision rule for a test gesture is designed. Dynamic time warping (DTW) has shown good performance on the simple time-series pattern recognition problems. The main drawback of this approach is the necessity to find the most appropriate samples to be included in a gesture class while keeping the number of these samples as low as possible to minimize the processing time. Wilson et al. compared the performance of Linear Time Warping and Dynamic Time Warping (DTW) to the HMM-based strategy in [26]. Liu et al. modified the DTW strategy to get more successful results [17].

\section{Proposed System}

\subsection{Detection of Activity Periods}

Traditional gesture recognition algorithms based on template matching consist of gesture detection and template matching. The first step is to collect a set of segments of time series per gesture class. Each segment of time series is called a template. In this paper, a collection of template time series corresponding to all gestures is called a gesture dictionary. The gesture recognition process aims to find the gesture class to which the most similar segment belongs by measuring the similarity between the input segment and all the templates in the dictionary. 
It is essential to accurately distinguish the gesture period from the training time series data in the process of collecting multiple templates per class. Detecting the start point and the end point of a gesture segment is called the detection of gesture period. If there is a pause interval between two gesture intervals, the gesture period and the non-gesture period can be easily distinguished, relatively, by comparing the absolute value of the signal sample with the threshold value. However, this method often causes an error in recognizing one long gesture as two or more short gestures when there is an interval within the gesture having characteristics similar to the pause period. A dynamic windowing capture method is also used to overcome these drawbacks [17].

Figure 1 compares the result of detecting the gesture period by applying the simple threshold comparison method [18] and the dynamic windowing capture method [17] to one template of the 14th gesture class among the basic gestures defined in this paper.

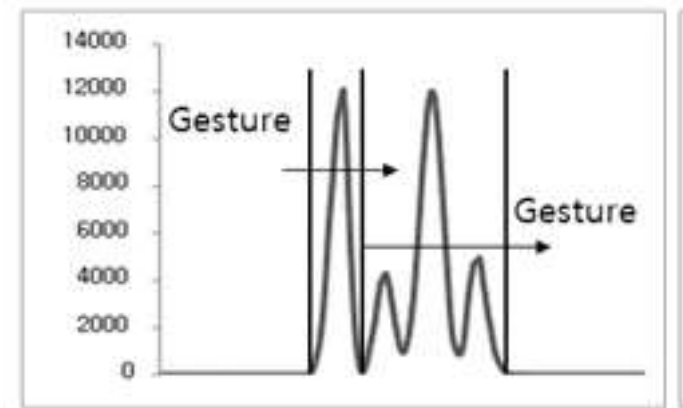

(a)

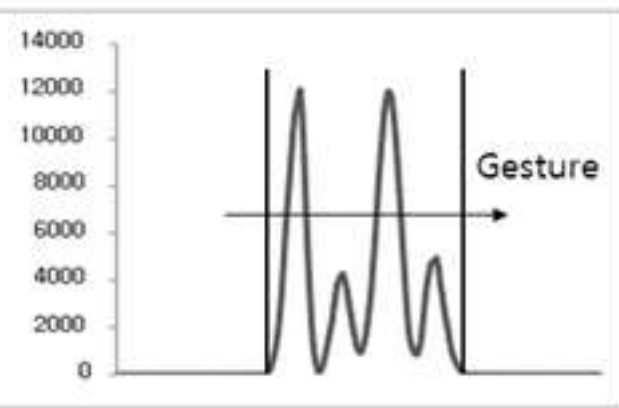

(b)

Figure 1. Comparative Results of Detecting the Gesture Periods: (a) the Simple Threshold Comparison Method, (b) the Dynamic Windowing Capture Method

In the graph in Figure 1, the horizontal axis is the time axis while the vertical axis is the sum of the magnitude of the three-axis gyroscope sensor values. Figure 1 (a) shows that the simple threshold comparison method detects two activation periods in one gesture interval while Figure 1 (b) shows the result of detecting the activation period of the gesture correctly by the dynamic windowing capture method. However, the dynamic windowing capture method can detect activation periods correctly only when the pause period before and after the gesture interval is secured. Moreover, this method cannot distinguish each gesture period when two gestures are continuously connected without pause period. For this reason, even though the dynamic windowing capture method is highly useful for automating the learning data collection process, it cannot be used for actual gesture recognition. In this paper, the dynamic windowing capture method is used only for the template data collection process

\subsection{Collection of Template Data}

The raw data to represent the gesture are a time series collected from the acceleration sensor and the gyroscope sensor attached to the user's lower arm. The raw data are collected while experimenters attaching the sensor to the lower arm repeat the predefined primitive gestures and pause postures several times. The analog signals from the two sensors are converted to digital data through an analog to digital converter (ADC) with a sampling frequency of $100 \mathrm{~Hz}$ and a resolution of 10 bits.

Let $a(t)=\left[\begin{array}{lll}a_{x}(t) & a_{y}(t) & a_{z}(t)\end{array}\right]^{T}$ be the signal of the acceleration sensor acquired at time $\mathrm{t}$, where $a_{x}(t), a_{y}(t), a_{z}(t)$ are the acceleration signals in three directions, respectively. Let $a(t)=\left[g_{x}(t) g_{y}(t) g_{z}(t)\right]^{T}$ be the signal of the gyroscope sensor 
acquired at time $\mathrm{t}$, where $g_{x}(t), g_{y}(t), g_{z}(t)$ are the angular velocity signals in three directions, respectively.

Let the sampling frequency of the $\mathrm{ADC}$ be $f_{s}$ and the sampling period be $T_{s}=1 / f_{s}$. The digital signals measured by the acceleration sensor and the gyroscope sensor are expressed as follows:

$$
a[n]=\left.a(t)\right|_{t=n T_{s}}
$$

and

$$
g[n]=\left.g(t)\right|_{t=n T_{-} s} .
$$

In this paper, we apply the dynamic windowing capture method proposed in [*] to the magnitude signal of the angular velocity component, $|g[n]|$, defined as follows to detect the activation interval of the gesture.

$$
|g[n]|=\sqrt{g_{x}^{2}[n]+g_{y}^{2}[n]+g_{z}^{2}[n]} .
$$

\subsection{Clustering Training Data}

Let us define $x[n]$ as follows using the signals of (1) and (2).

$$
x[n]=\left[\begin{array}{l}
a[n] \\
g[n]
\end{array}\right]
$$

Let $N$ be the number of different gestures and let $\left\{X_{i j}: 1 \leq j \leq M\right\}$ be the set of acceleration and angular velocity sample vectors obtained by repeating the $i$-th gesture $M$ times each.

$$
X_{i j}=\left[x_{i j}[0], x_{i j}[1], \cdots, x_{i j}\left[v_{i j}-1\right]\right] .
$$

Even for the same user, the length of the activation period may be different each time the same gesture is repeated. Since the dynamic ranges of the acceleration sensor and the gyroscope sensor are different from each other, the data values of the gyroscope sensor and the acceleration sensor are normalized to the range between $[0,1]$. Let $X=$ $\left\{x \in X_{i j}: 1 \leq i \leq N, 1 \leq j \leq M\right\}$. Subsequently, the number of samples $\mathrm{L}$ used for clustering is as follows.

$$
L=\sum_{i=1}^{N} \sum_{j=1}^{M} v_{i j}
$$

All samples included in $X$ are clustered using the $k$-means clustering algorithm [16] and each sample is assigned to one of the $K$ different clusters $S_{k} 1 \leq k \leq K$.

Let $S_{k}$ be the $k$-th cluster and, let $S=\left\{S_{1}, S_{2}, \cdots S_{K}\right\}$. If we let the index set of the cluster be $I=\{k: 1 \leq k \leq K\}$, and the central coordinate of the cluster $S_{k}$ be $\mu_{k}, k \in I$, then the set $S$ determined by the $k$-means clustering algorithm is determined as follows.

$$
\underset{S}{\operatorname{argmin}} \sum_{k=1}^{K} \sum_{x \in S_{k}}\left\|x-\mu_{k}\right\|^{2}
$$

Let $G=\left\{g_{i j}: 1 \leq i \leq N, 1 \leq j \leq M\right\}$ be the gesture dictionary, where 


$$
\begin{aligned}
& g_{i j}=\left[k_{i j}[0], k_{i j}[1], \cdots, k_{i j}\left[v_{i j}-1\right]\right] . \\
& \quad \text { Meanwhile, } k_{i j}[n], 0 \leq n \leq v_{i j}-1 \text { satisfies the following equation. } \\
& k_{i j}[n]=\underset{k}{\operatorname{argmin}}\left|x_{i j}[n]-\mu_{k}\right|^{2} .
\end{aligned}
$$

\subsection{The Gesture Recognition Algorithm}

One may construct $X$ by collecting consecutive $P$ samples from the input signal as follows: $\quad X[n]=[x[n] x[n+1] \cdots x[n+P-1]]^{T}, \quad$ where $\quad P=\max \left(v_{i j} ; 1 \leq i \leq\right.$ $N ; 1 \leq j \leq M)$. Let $k[n]=\underset{k}{\operatorname{argmin}}\left|x[n]-\mu_{k}\right|$ and define the index vector as $K[n]=$ $[k[n] k[n+1] \cdots k[n+P-1]]^{T}$. The gesture recognition problem of the current signal $X[n]$ can be expressed as follows.

First, for each gesture $i, 1 \leq i \leq N$, the index $j_{i}$ of $X_{i}$ with the smallest error in the gesture dictionary is obtained.

$j_{i}=\underset{j}{\operatorname{argmin}} d\left(X[n]-X_{i j}\right)$, for $i \in I$.

As explained above, it is not easy to obtain $d\left(X[n]-X_{i j}\right)$ in Equation (10) because the lengths of $X_{i j}$ are not all the same and the activation period of the signal $X[n]$ to be recognized is not accurately distinguished. In this paper, only the first samples are used to calculate the distances so that $d\left(X[n]-X_{i j}\right)$ is equal to the activation period length $v_{i j}$ of $X_{i j}$ in $X[n]$. The distance function used in this paper is defined as follows.

$$
d\left(X[n]-x_{i j}\right)=\frac{1}{v_{i j}} \sum_{v=0}^{v_{i j}-1}\left\{\begin{array}{cr}
0, & \text { if } k[n+v]=k_{i j}[v] \\
\left\|x[n+v]-\mu_{i j}\right\|, \text { if } k[n+v] \neq k_{i j}[v]
\end{array}\right.
$$

It is noted that the recognition rate is better in real experiments when the distance between the $x$ and $x_{i j}$ cluster indices is set to 0 . The gestures obtained through this process are called candidate gestures. Let us now define the error signals composed of the mean error of each candidate gesture as follows.

$e[n]=\left[e_{1}[n] \quad e_{2}[n] \cdots e_{N}[n]\right]^{T}$,

where, $e_{i}[n]=d\left(X[n]-X_{i_{i}}[n]\right)$. In selecting the final gesture among the candidate gestures, when a gesture having a minimum value of $e_{i}[n]$ is simply selected, a wrong selection often occurs when there is not much difference in errors between different candidate gestures. In this case, judge $X[n]$ as a gesture one sample before. In this process, the range of the value of $e_{i}[n]$ varies greatly as $n$ changes. To prevent this phenomenon, the Mahalanobis distance is used to normalize the error.

The same method is applied to $\mathrm{X}[\mathrm{u}]=[x[u] x[u+1] \cdots x[u+P-1]]^{T}, u \geq 1$ to repeat the gesture recognition by sample. The algorithm proposed in this paper is divided into forward search and backward search algorithms according to the direction of the template search. When a gesture candidate is determined after the forward search is performed, the backward search is performed. The backward search start position is the start position of the forward search plus the gesture candidate length. If the backward search result is the same as the forward one, the forward search starts again after the recognized gesture period. 


\section{Experiments and Discussion}

\subsection{Implementation of Experimental Apparatus}

In this paper, the signals used for gesture recognition are three- axis acceleration signal combined with three- axis gyroscope signal. We implemented a wristwatch type experiment device and software to collect the raw data representing the gesture. The MCU used in the implementation of the wristwatch type experiment device is Arduino Pro mini 3.3v / 8M, a small Arduino version using the ATMEGA328P chip, with a 6-axis IMU sensor MPU6050 being used. In order to minimize the restriction of arm movement by the wired connector, the sensor signals were transmitted wirelessly through the Bluetooth module of the HC-06 model, and a lithium battery was used as the power source. Figure 2 shows the data collection device implemented.

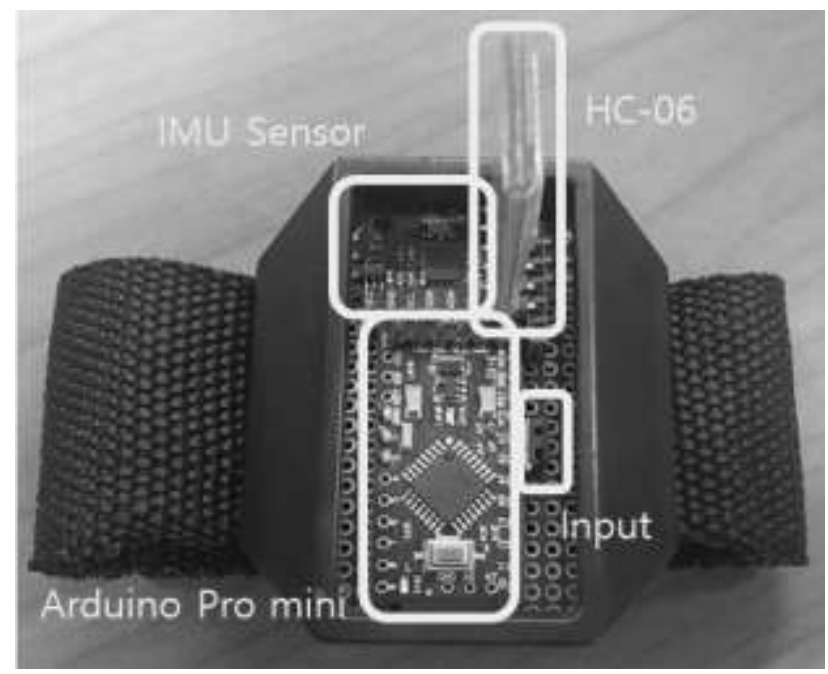

\section{Figure 2. Data Collection Device Implemented}

We have implemented a data collection program in the Python programming language, which runs on Microsoft Windows 10. The data acquisition device continuously transmits the three-axis acceleration signal and the three-axis gyroscope signal to the PC through the Bluetooth wireless channel, while the data acquisition program can collect data through a simple key operation.

\subsection{Template Data Collection}

In this paper, 14 primitive gestures are defined. Table 1 lists the primitive gestures defined in this paper.

The training data collection procedure is as follows. The data collecting device starts collecting the data in the ready position, the experimenter performs a primitive gesture, and the data collecting device collects a pause signal for a certain period of time at the end of the action. This procedure is repeated several times for each gesture. Additional signals with no motion in the ready position are also collected. A total of 560 primitive gesture templates, 40 for each 14 gestures, per experimenter, were collected. A total of 4 experimenters participated in the data collection and 2240 primitive gesture templates were collected. A gesture dictionary was constructed by randomly selecting 32 templates per gesture out of 2240 templates. In other words, a gesture dictionary with 448 templates was created, and the remaining 1792 were used for testing. 
Table 1. The 14 Primitive Hand Gestures Defined in this Paper

\begin{tabular}{|c|c|c|c|c|}
\hline $\begin{array}{l}\text { Ready } \\
\text { Posture }\end{array}$ & $\begin{array}{l}\text { Arm's } \\
\text { movement of } \\
\text { gesture }\end{array}$ & $\begin{array}{l}\text { Gesture } \\
\text { number }\end{array}$ & $\begin{array}{l}\text { Arm's } \\
\text { movement of } \\
\text { gesture }\end{array}$ & $\begin{array}{l}\text { Gesture } \\
\text { number }\end{array}$ \\
\hline & & 1 & & 2 \\
\hline & & 3 & & 4 \\
\hline & & 5 & & 6 \\
\hline & & 7 & & 8 \\
\hline & & 9 & & 10 \\
\hline & & 11 & & 12 \\
\hline & & 13 & & 14 \\
\hline
\end{tabular}

\subsection{Performance Comparison of the Algorithms}

Table 2 shows the resulting confusion matrix of the proposed method. The number of test gestures is 1790 . The overall recognition accuracy on the test set is $98.64 \%$.

Figure 3 compares the recognition accuracy on the multiple concatenated gestures, each of which consists of five gestures randomly selected out of 14 gestures. In the graph of Figure 4, the abscissa represents the ratio of the period of the gesture with the shortest gesture out of the five gestures to the period of the pause between the gestures. Pause data of the same length was added after the last gesture. In Figure 4, M1 means forward search method and M2 means bidirectional search method.

In Figure 3, DTW [17] and HMM [25] show high performance for single gesture recognition with relatively long pause, but gesture recognition performance decreases as the gesture interval becomes smaller.

Table 2. A Confusion Matrix for 14 Hand Gestures

\begin{tabular}{|l|l|l|l|l|l|l|l|l|l|l|l|l|l|l|}
\hline & $\mathrm{g} 1$ & $\mathrm{~g} 2$ & $\mathrm{~g} 3$ & $\mathrm{~g} 4$ & $\mathrm{~g} 5$ & $\mathrm{~g} 6$ & $\mathrm{~g} 7$ & $\mathrm{~g} 8$ & $\mathrm{~g} 9$ & $\mathrm{~g} 10$ & $\mathrm{~g} 11$ & $\mathrm{~g} 12$ & $\mathrm{~g} 13$ & $\mathrm{~g} 14$ \\
\hline $\mathrm{g} 1$ & 128 & & & & & & & & & & & & & 1 \\
\hline $\mathrm{g} 2$ & & 127 & & & & & & & & & & & & \\
\hline $\mathrm{g} 3$ & & & 128 & 1 & & & & & & 1 & & & & \\
\hline $\mathrm{g} 4$ & & & & 127 & & & & & & 1 & & & & 1 \\
\hline $\mathrm{g} 5$ & & 1 & & & 124 & & 2 & & & & & & 1 & \\
\hline $\mathrm{g} 6$ & & & & & & 125 & 1 & 2 & & & & & & \\
\hline $\mathrm{g} 7$ & & & & & 2 & & 125 & & & & & & 2 & \\
\hline $\mathrm{g} 8$ & & & & & 2 & 3 & & 125 & & & & & & \\
\hline $\mathrm{g} 9$ & & & & & & & & 1 & 126 & & & & & \\
\hline $\mathrm{g} 10$ & & & & & & & & & 2 & 126 & & & & \\
\hline
\end{tabular}




\begin{tabular}{|l|l|l|l|l|l|l|l|l|l|l|l|l|l|l|}
\hline $\mathrm{g} 11$ & & & & & & & & & & & 128 & & & \\
\hline $\mathrm{g} 12$ & & & & & & & & & & & & 128 & & \\
\hline $\mathrm{g} 13$ & & & & & & & & & & & & & 125 & \\
\hline $\mathrm{g} 14$ & & & & & & & & & & & & & & 126 \\
\hline
\end{tabular}

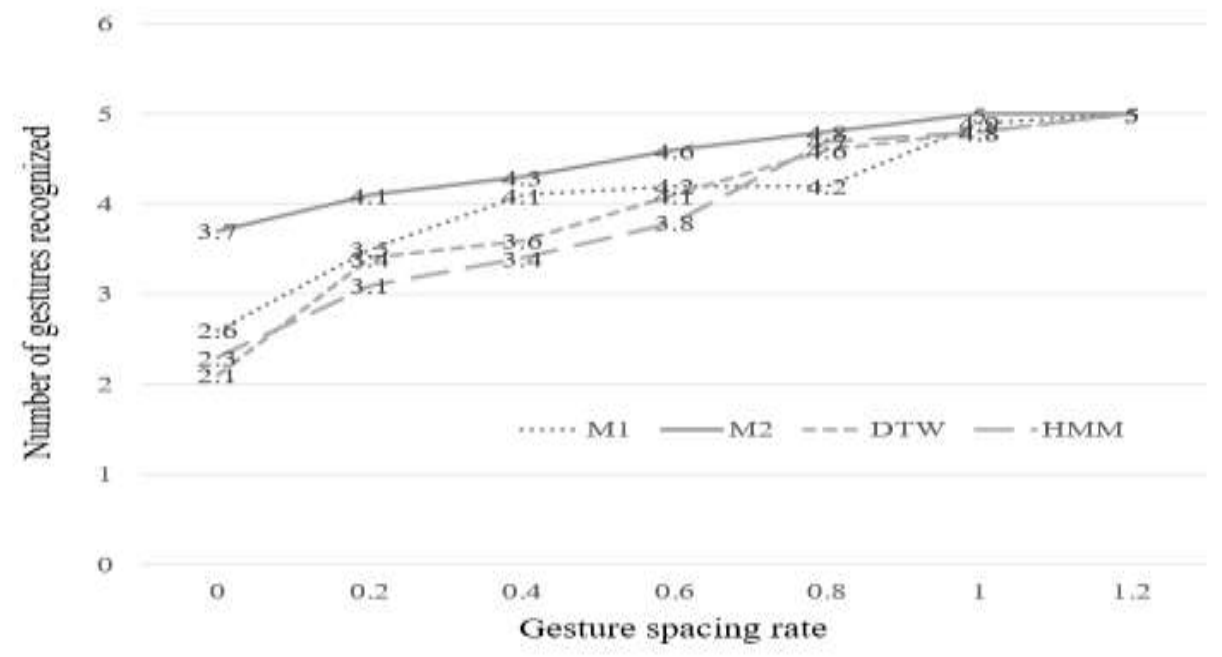

Figure 3. Recognition Rate According to Gesture Spacing Rate

\section{Conclusion}

In this paper, a dictionary-based template matching algorithm that is capable of recognizing multiple concatenated gestures without detecting the activity period in advance is proposed. The proposed algorithm is applied to a three-axis gyroscope sensor signal combined with a three-axis acceleration sensor signal.

The raw data is collected while experimenters attaching the sensor to the lower arm repeat the predefined primitive gestures and pause postures several times. The samples of the collected time series are clustered in a six-dimensional vector space, while each cluster index and center coordinates are obtained and a gesture dictionary is constructed by collecting them. After applying the variable-length sliding window to the input time series signal and calculating the error by comparing with the templates in the dictionary, the gesture with the smallest error was selected as the target gesture. A wrong selection often occurs when there is not much difference in errors between different candidate gestures. To reduce such recognition errors, the error is normalized by the Mahalanobis distance.

In order to evaluate the performance of the proposed algorithm, experimentation was done on changing the period of the pause interval between two consecutive gestures. As a result of 14 primitive gesture recognition tests with the proposed algorithm, the overall recognition accuracy of $96 \%$ or more was obtained regardless of the length of the pause period between two adjacent gestures.

The conventional sensor-based gesture recognition method is generally composed of two steps: the detection of the activity period followed by the recognition of the detected sensor signal segment. If there is no pause period between two consecutive gestures or if it is short, it is difficult to detect the activity period, which in turn causes the degradation of the gesture recognition accuracy.

The recognition rate of the proposed algorithm depends on the type of gesture, the number of gestures, and the number of clusters. In the future, the relationship among the number of basic gestures, the complexity of gestures, and the number of clusters will be studied. 


\section{Acknowledgments}

This research was supported by Hallym University Research Fund (HRF-201706-013). This paper is a revised and expanded version of a paper entitled "continuous arm gesture recognition algorithm using clustering of wearable inertial sensor signals" presented at the 13-th Interdisciplinary Workshop 2017, Jeju, Korea, Aug. 2017.

\section{References}

[1] C. Yang, David K. Han and H. Ko, "Continuous hand gesture recognition based on trajectory shape information", Pattern Recognition Letters, vol. 99, (2017), pp. 39-47.

[2] S. Mitra and T. Acharya, "Gesture recognition: A survey", IEEE Transactions on Systems, Man, and Cybernetics, Part C (Applications and Reviews), vol. 37. no. 3, (2007) pp. 311-324.

[3] H. Hasan and S. Abdul-Kareem, "Retraction Note to: Human-computer interaction using vision-based hand gesture recognition systems: a survey", Neural Computing and Applications, vol. 28, no. 4, (2017), pp. 849-849.

[4] H. Cheng, L. Yang and Z. Liu, "Survey on 3D hand gesture recognition", IEEE Transactions on Circuits and Systems for Video Technology, vol. 26, no. 9, (2016), pp. 1659-1673.

[5] K. Nymoen, M. R. Haugen and A. R. Jensenius, "MuMYO-Evaluating and Exploring the MYO armband for musical interaction", Proceedings of the international conference on New Interfaces for Musical Expression, Louisiana, USA, (2015) May 31-June 3.

[6] F. Kerber, M. Puhl and A. Krüger, "User-Independent Real-Time Hand Gesture Recognition Based on Surface Electromyography", Proceedings of the 19th International Conference on Human-Computer Interaction with Mobile Devices and Services, Vienna, Austria, (2017), September 04-07.

[7] S. Shin, D. Kim and Y. Seo, "Controlling Mobile Robot Using IMU and EMG Sensor-Based Gesture Recognition", Proceedings of the IEEE Ninth International Conference on the Broadband and Wireless Computing, Communication and Applications (BWCCA), 2014, Guangdong, China, (2014), pp. November 8-14.

[8] S. Shin, J. Lee and S. H. Son, "A Survey of Approaches for Recognizing Hand Gestures Using EMG Signal", Proceedings of the 22nd IEEE International Conference on Embedded and Real-Time Computing Systems and Applications, Daegu, Korea, (2016), August 17-19.

[9] A. Srisuphab and P. Silapachote, "Artificial neural networks for gesture classification with inertial motion sensing armbands", Proceedings of the IEEE Region 10 Conference (TENCON), Singapore, (2016), November 22-25.

[10] X. Chen, X. Zhang, Z. Y. Zhao, J. H. Yang, V. Lantz and K. Q. Wang, "Hand gesture recognition research based on surface EMG sensors and 2D-accelerometers", Proceedings of the 2007 11th IEEE International Symposium on Wearable Computers, Boston, MA, USA, (2007) October 11-13.

[11] Kinect for Windows Sensor Components and Specifications (accessed September 30, 2017), Retrieved from https://msdn.microsoft.com/en-us/library/jj131033.aspx.

[12] Myo Tech Specs, (accessed September 30, 2017), Retrieved from https://www.myo.com/techspecs.

[13] P. Premaratne, "Human computer interaction using hand gestures", Springer Science \& Business Media, (2014).

[14] P. Henry, M. Krainin, E. Herbst and D. Fox, "RGB-D Mapping: Using Depth Cameras for Dense 3D Modeling of Indoor Environments", Proceedings of the 12th International Symposium on Experimental Robotics, Delhi, India, (2010) December 18-21.

[15] X. Zhang, X. Chen, Y. Li, V. Lantz, K. Wang and J. Yang, "A framework for hand gesture recognition based on accelerometer and EMG sensors", IEEE Transactions on Systems, Man, and Cybernetics-Part A: Systems and Humans 41.6, (2011). pp. 1064-1076.

[16] J. Taylor, "Real-time translation of American Sign Language using wearable technology", (2016), Honors Theses, 928, https://scholarship.richmond.edu/honors-theses/928.

[17] H. P. Gupta, H. S. Chudgar, S. Mukherjee, T. Dutta, and K. Sharma, "A Continuous Hand Gestures Recognition Technique for Human-Machine Interaction Using Accelerometer and Gyroscope Sensors", IEEE Sensors Journal, vol. 16, no. 16, (2016), pp. 6425-6432.

[18] C. Keskin, A. T. Cemgil, and L. Akarun, "DTW Based Clustering to Improve Hand Gesture Recognition", Proceedings of the Second International Workshop on Human Behavior Understanding: Inducing Behavioral Change, Amsterdam, The Netherlands, (2011) November 16-18.

[19] F. Hofmann, P. Heyer and G. Hommel, "Velocity profile based recognition of dynamic gestures with discrete hidden markov models", Proceedings of the International Gesture Workshop on Gesture and Sign Language in Human-Computer Interaction, Bielefeld, Germany, (1997) September 17-19.

[20] S. Kallio, J. Kela and J. Mantyjarvi, "Online gesture recognition system for mobile interaction," Proceedings of the IEEE International Conference on Systems, Man and Cybernetic, Washington, DC, USA, (2003) October 8.

[21] T. Pylvänäinen, "Accelerometer based gesture recognition using continuous HMMs", Proceedings of the 2nd Iberian Conference on Pattern Recognition and Image Analysis, Estoril, Portugal, (2005) June 7-9. 
[22] V. M. Mantyla, J. Mantyjarvi, T. Seppanen and E. Tuulari, "Hand gesture recognition of a mobile device user", Proceedings of the IEEE International Conference on Multimedia and Expo, New York, NY, USA, (2000) July 30-August 2.

[23] B. W. Choe, J. K. Min and S. B. Cho, "Online gesture recognition for user interface on accelerometer built-in mobile phones", International Conference on Neural Information Processing Neural Information Processing. Models and Applications, Sydney, NSW, Australia, (2010) November 22-25.

[24] M. Hoffman, P. Varcholik and J. J. LaViola. "Breaking the status quo: Improving 3D gesture recognition with spatially convenient input devices," Proceedings of the 2010 IEEE Virtual Reality Conference (VR), Waltham, Massachusetts, USA, (2010) March 20-26.

[25] G. Lefebvre, S. Berlemont, F. Mamalet and C. Garcia, "BLSTM-RNN based 3D gesture classification", Proceedings of the International Conference on Artificial Neural Networks, Sofia, Bulgaria, (2013), September 10-13.

[26] D. H. Wilson and A. Wilson. "Gesture recognition using the XWand.", Technical Report CMU-RI-TR04-57, Robotics Institute, April, Retrieved from http://repository.cmu.edu/robotics/729/, (2004).

\section{Authors}

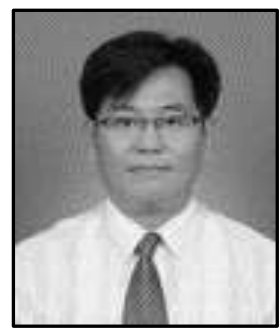

Byeong Jeong Kim, he received the B.S and M.A and Ph.D(c) from Hallym University and Hanyang University, Korea, in 1999, 2001 and 2011, respectively, all in electrical engineering. From 2001 to 2007, he has worked in the Video Transmission and Enhancement Technology. He joined Hallym University, Gangwon-do, Korea, in September 2007, where he is currently an Assistant Professor in the Department of Electronic Engineering.

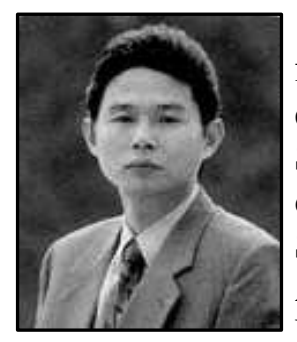

Jae-il Jung, he received the B.S. degree in electronic engineering from Hanyang University, Seoul, Korea, in 1981, M.S. degree in electrical and electronic engineering from Korea Advanced Institute of Science and Technology (KAIST), Seoul, Korea, in 1984, and Ph.D. degree in computer science and networks from Ecole Nationale Superieure des Telecommunica-tions (ENST), Paris, France, in 1993. After receiving his M.S. degree, he was with Telecommunication Network Research Labs., Korea Telecom, from 1984 to 1997. He is currently a professor at Hanyang University. His research interests include broadband networks and services, switching systems, especially in wireless networks, and communications.

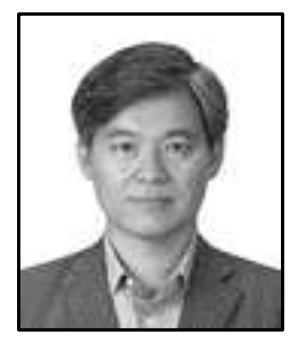

Seop Hyeong Park, he received the B.S., M.A., and Ph.D. degrees from Seoul National University, Korea, in 1984, 1986, and 1990, respectively, all in electrical engineering. From 1990 to 1992, he was with the HDTV Development Center, Korean Academy of Industrial Technology. From 1992 to 1998, he was with Telecommunication Network Research Labs., Korea Telecom. In 1993, he was a visiting researcher at the NTT Human Interface Laboratory, Yokosuka, Japan. He joined Hallym University, Gangwon-do, Korea, in March 1998, where he is currently a Professor in the Department of Electronic Engineering. In 2004, he was a visiting scholar at the University of California, Santa Barbara. From 2006 to 2009, he was the dean of College of Information and Electronics, Hallym University. His research interests are signal processing including speech, video and ultrasound signals, multimedia communication, augmented reality, pattern recognition and machine learning. 
International Journal of Control and Automation

Vol. 11, No. 5 (2018) 